\title{
Towards Enhanced Electrochemical Capacitance with Preparation of La-doped $\mathrm{MnO}_{2}$ Nanoparticles
}

\author{
Naying Zhao ${ }^{1, a}$, Zhenzhong Hou ${ }^{1, b}$, Qinghao Yang ${ }^{1, c}$ and Xuejian Yang ${ }^{2, d}$ \\ ${ }^{1}$ College of materials science and Engineering, Xi'an University of Science and Technology, Xi'an, \\ China, 710054 \\ ${ }^{2}$ College of Safety Science and Engineering, Xi'an University of Science and Technology, Xi'an, \\ China, 710054

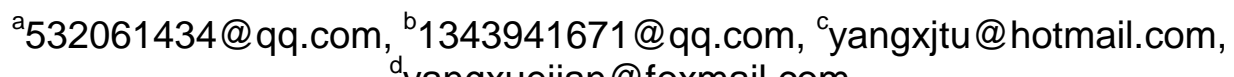 \\ yangxuejian@foxmail.com
}

Keywords: manganese dioxide, nanoparticles, supercapacitance, sol-gel method Abstract. La-doped $\mathrm{MnO}_{2}$ nanoparticles were synthesized by a facile sol-gel method. Using XRD and SEM to analyze the structure and morphology of the obtained nano- $\mathrm{MnO}_{2}$, the results show that it exhibit $\alpha-\mathrm{MnO}_{2}$ crystal form with some amorphous structure and irregular granular morphology with size of 150-300 nm. Through electrochemical tests of CV and GCD, La-doped nano- $\mathrm{MnO}_{2}$ presents enhanced electrochemical capacitance with a specific capacitance of $168.7 \mathrm{~F} \mathrm{~g}^{-1}$, much higher than that of pure nano- $\mathrm{MnO}_{2}$ prepared at the same conditions.

\section{Introduction}

As well as we know, the super-capacitor with combining the advantages of batteries and capacitors has high power density and long service life, becoming a research hotspot in the world ${ }^{[1-2]}$. In high performance battery, electrolytic manganese dioxide $\left(\mathrm{MnO}_{2}\right)$ has been a kind of indispensable raw material ${ }^{[3]}$. Recently years, many researchers have applied themselves to investigating the preparation and properties of nano- $\mathrm{MnO}_{2}$ so as to overcome the disadvantages of the electrolytic manganese dioxide (EMD) including long production period, high energy consumption and high cost. Sol-gel method is an effective and environment-friendly approach to prepared nano- $\mathrm{MnO}_{2}$, while avoiding the aboved disadvantages. It was reported that nano- $\mathrm{MnO}_{2}$ as electrode materials could significantly improve the capacitance ${ }^{[4,5]}$. Wei $\mathrm{H}$ and colleagues ${ }^{[6]}$ have prepared one-dimensional nanostructured $\alpha-\mathrm{MnO}_{2}$, which is a promising candidate of electrode materials for high effective supercapacitors, via facile hydrothermal synthesis. This nano- $\alpha-\mathrm{MnO}_{2}$ exhibits a specific capacitance of $158 \mathrm{~F} \mathrm{~g}^{-1}$ at current density of $4 \mathrm{~A} \mathrm{~g}^{-1}$ and fine cycle stability. Yu Y. et al. ${ }^{[7]}$ have synthesized the ultrathin $\mathrm{MnO}_{2}$ nanosheets with a thickness of $0.9 \mathrm{~nm}$ for flexible supercapacitors, which presents a specific capacitance of $98 \mathrm{~F} \mathrm{~g}^{-1}$ at $50 \mathrm{~A} \mathrm{~g}^{-1}$ and a capacitance retention after 6000 cycles of $95 \%$. In this work, we have fabricated La-doped $\mathrm{MnO}_{2}$ nanoparticles by sol-gel method and investigated its electrochemical capacitance.

\section{Experiment}

Synthesis. All the reagents were analytical pure and used as received. The La-doped $\mathrm{MnO}_{2}$ nanoparticles were prepared by sol-gel method. A typical preparation process is as follows: citric acid $\left(\mathrm{C}_{6} \mathrm{H}_{8} \mathrm{O}_{7}, 5.2535 \mathrm{~g}\right)$, manganous acetate $\left[\mathrm{Mn}\left(\mathrm{CH}_{3} \mathrm{COO}\right)_{2}, 12.2545 \mathrm{~g}\right]$ and appropriate lanthanum nitrate $\left[\mathrm{La}\left(\mathrm{NO}_{3}\right)_{3}\right]$ were firstly dissolved in deionized water to form a uniform solution. Then a small amount of ammonia water was added to adjust the $\mathrm{pH}$ value to 6 , consequently the sol was formed at $80{ }^{\circ} \mathrm{C}$ via a liquid phase chemical reaction. After drying, the sol changed into a gel, which has an open skeleton structure. Finally, the products of La-doped $\mathrm{MnO}_{2}$ nanoparticles were obtained during the high temperature calcination. In order to further improve the conductivity, La-doped $\mathrm{MnO}_{2}$ 
nanoparticles were added into sulfuric acid solution for $2 \mathrm{~h}$, then the as-prepared sample was filtered and washed with ethanol and deionized water for several times until the filtrate became neutral. After drying, the obtained solid powder is acid treated La-doped $\mathrm{MnO}_{2}$ nanoparticles. As the control sample, the pure $\mathrm{MnO}_{2}$ nanoparticles were prepared following the same method above without adding $\mathrm{La}\left(\mathrm{NO}_{3}\right)_{3}$.

Characterization. XRD was carried out on a D/max 2550 spectrograph with a copper target at a scan speed of $6.00 \mathrm{deg} / \mathrm{min}$. The micro-morphology of prepared $\mathrm{MnO}_{2}$ nanoparticles was observed by SEM (JSM-6460LV, JEOL, Japan). Electrochemical performance of the samples were evaluated by cyclic voltammetry (CV) and galvanostatic charge/discharge (GCD), which were performed on CS 350 electrochemical workstation (Corrtest Corporation, Wuhan Chian) at room temperature. All electrochemical experiments were carried out in $1 \mathrm{~mol} / \mathrm{L} \mathrm{Na}_{2} \mathrm{SO}_{4}$ solution using a three-electrode system, which contained a saturated calomel electrode and a platinum plate (ca. $1 \mathrm{~cm}^{2}$ ) as the reference electrode and counter electrode, respectively, the obtained $\mathrm{MnO}_{2}$ nanoparticles loaded on foam nickel $\left(1 \mathrm{~cm}^{2}\right)$ as the working electrode. (Working electrodes were prepared by mixing $80 \mathrm{wt} \%$ as-prepared $\mathrm{MnO}_{2}$ with $10 \mathrm{wt} \%$ acetylene black and $10 \mathrm{wt} \%$ polytetrafluoroethylene dissolved in ethanol as a binder to form a homogeneous slurry. The obtained slurry was then pressed onto a foam nickel at $10 \mathrm{MPa}$ for $45 \mathrm{~s}$ and dried under vacuum at $60{ }^{\circ} \mathrm{C}$ for $24 \mathrm{~h}$.)

\section{Results and Discussion}

\section{Structure and Morphology of the $\mathrm{MnO}_{2}$ Nanoparticles.}

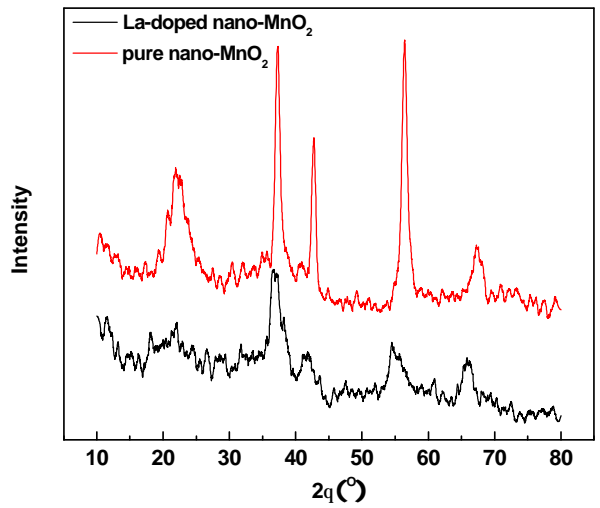

Fig. 1 XRD patterns of as-prepared $\mathrm{MnO}_{2}$ nanoparticles. Red line is pure nano- $\mathrm{MnO}_{2}$; black line is La-doped nano- $\mathrm{MnO}_{2}$.

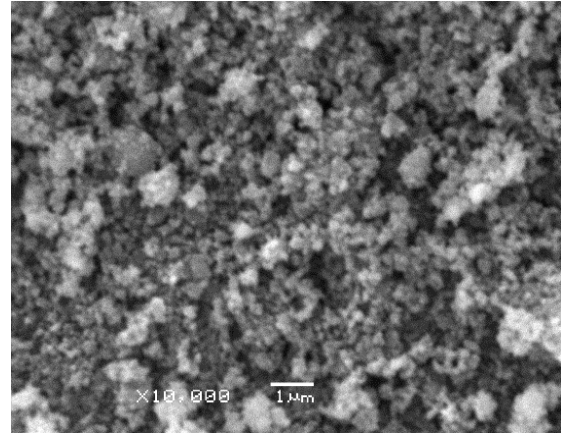

Fig. 2 SEM images of the La-doped $\mathrm{MnO}_{2}$ nanoparticles.

Figure 1 shows the XRD patterns of as-prepared pure $\mathrm{MnO}_{2}$ nanoparticles and La-doped $\mathrm{MnO}_{2}$ nanoparticles. It can be found that the two patterns have the same diffraction peaks at about $22^{\circ}, 38^{\circ}$, $42^{\circ}, 58^{\circ}$ and $68^{\circ}$, which belong to the characteristic peaks of $\alpha-\mathrm{MnO}_{2}$ according to the PDF standard card (440141) of XRD, indicating that the two kinds of nano- $\mathrm{MnO}_{2}$ have the same crystal, in other words, La-doping has no effect on crystal form of the obained $\mathrm{MnO}_{2}$. In addition, comparing with the pattern of pure $\mathrm{MnO}_{2}$ nanoparticles, the one of La-doped $\mathrm{MnO}_{2}$ nanoparticles exhibits weaker and broader diffraction peaks, revealing more amorphous in structure. It is seen from Figure 2 that the La-doped $\mathrm{MnO}_{2}$ nanoparticles with size of 150-300 nm gather mutually and exhibit an irregular granular morphology. As the electrode material for supercapacitor, La-doped nano- $\mathrm{MnO}_{2}$ with some amorphous structure could provide larger specific surface area and higher porosity than bulk high-crystallized $\mathrm{MnO}_{2}$, which allow fast ionic migration and charge storage within the material during the electrochemical reactions, favouring an improvement in electrochemical capacitance ${ }^{[8]}$. 
Electrochemical Capacitance of the $\mathrm{MnO}_{2}$ Nanoparticles.

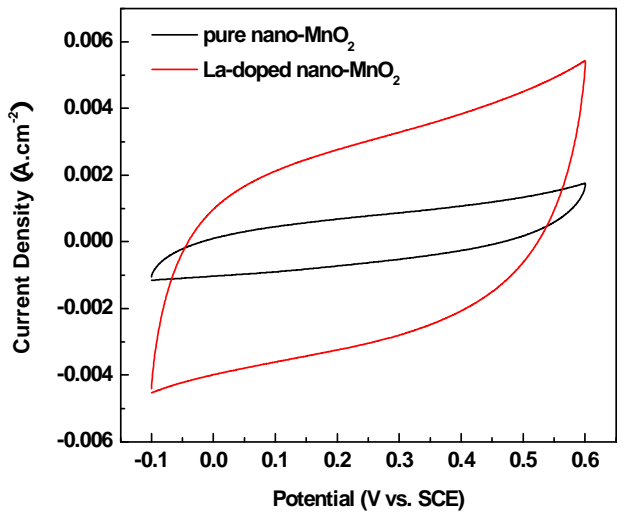

Fig. $3 \mathrm{CV}$ curves of pure and La-doped nano- $\mathrm{MnO}_{2}$ electrodes in $1 \mathrm{~mol} / \mathrm{L} \mathrm{Na}_{2} \mathrm{SO}_{4}$ with a sweep rate of $20 \mathrm{mV} \mathrm{s}^{-1}$ at $25^{\circ} \mathrm{C}$.

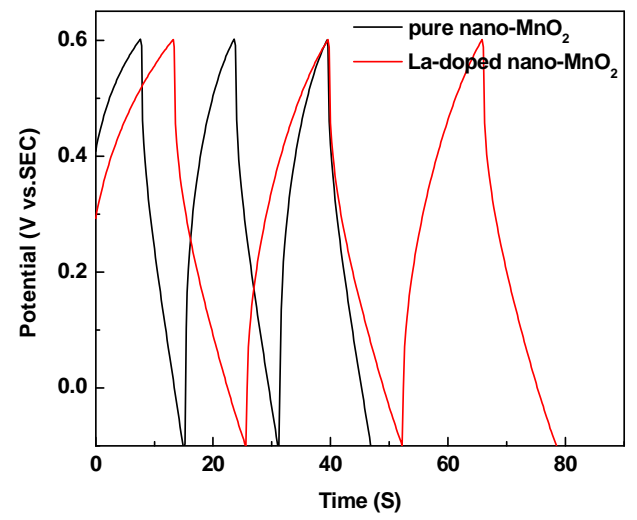

Fig. 4 GCD curves of pure and La-doped nano- $\mathrm{MnO}_{2}$ electrodes in $1 \mathrm{~mol} / \mathrm{L} \mathrm{Na}_{2} \mathrm{SO}_{4}$ at a current density of $0.3 \mathrm{~A} \mathrm{~g}^{-1}$.

Figure 3 depicits the $\mathrm{CV}$ curves of pure and La-doped nano- $\mathrm{MnO}_{2}$ electrodes in $1 \mathrm{~mol} / \mathrm{L} \mathrm{Na}_{2} \mathrm{SO}_{4}$ solution at $20 \mathrm{mV} \mathrm{s}^{-1}$. Obviously, both curves in this figure show a typical faradaic capacitance performance with symmetrical redox behavior, and the current response of La-doped nano- $\mathrm{MnO}_{2}$ is evidently larger, suggesting its enhanced electrochemical capacitance. According to the equation of $C=\int \frac{I}{v m V} d V^{[9]}$,Where $C$ is the specific capacitance $\left(\mathrm{F} \mathrm{g}^{-1}\right) ; I$ is the response current $(\mathrm{A}) ; m$ is the the mass of the electroactive material in the electrode $(\mathrm{g}) ; v$ is the potential scan rate $\left(\mathrm{V} \mathrm{s}^{-1}\right)$ and $V$ is the potential $(\mathrm{V})$, the specific capacitance of La-doped nano- $\mathrm{MnO}_{2}$ with acidification can be calculated as $168.7 \mathrm{~F} \mathrm{~g}^{-1}$, more higher than that of pure nano- $\mathrm{MnO}_{2}\left(76 \mathrm{~F} \mathrm{~g}^{-1}\right)$. The similar result is also confirmed by GCD measurements, as shown in Figure 4. The La-doped nano- $\mathrm{MnO}_{2}$ electrode exhibit longer discharge time at the same current densities, implying its improved specific capacitance ${ }^{[10]}$.

\section{Conclusions}

The La-doped $\mathrm{MnO}_{2}$ nanoparticles have been prepared by a facile sol-gel method. The obtained nanoparticles exhibit $\alpha-\mathrm{MnO}_{2}$ crystal form with some amorphous structure and irregular granular morphology with size of $150-300 \mathrm{~nm}$, as demonstrated by XRD and SEM, respectively. After acidification, the La-doped nano- $\mathrm{MnO}_{2}$ delivers a much higher specific capacitance of $168.7 \mathrm{~F} \mathrm{~g}^{-1}$ than pure nano- $\mathrm{MnO}_{2}$ synthesized at the same conditions. These results indicate the La-doped nano- $\mathrm{MnO}_{2}$ possesses enhanced electrochemical performance, just being appropriate for supercapacitor application.

\section{Acknowledgments}

This work was financially supported by National Natural Science Foundation of China(21204072) and Key Scientific and Technological innovation team Program of Shaanxi Province, China (2014K CT-04).

\section{References}

[1] Largeot C, Portet C, Chmiola J, et al: Journal of the American Chemical Society. 130(2008), p. 2730.

[2] Kandalkar SG, Dhawale DS, Kim CK, et al: Synthetic Metals. 160(2010), p. 1299.

[3] Li Tongqing: Battery Bimonthly. 31(2001), p. 52.

[4] Srither SR, Karthik A, Arunmetha S, et al: Materials Chemistry and Physics. 183(2016), p. 375. 
[5] Dang W, Dong C, Zhang Z, et al: Electrochimica Acta. 217(2016), p. 16.

[6] Wei H, Wang J, Yang S, et al: Physica E: Low-dimensional Systems and Nanostructures. 83(2016), p. 41.

[7] Yu Y, Zhai Y, Liu H, et al: Materials Letters. 176(2016), p. 33.

[8] Shi Y, Pan L, Liu B, et al: Journal of Materials Chemistry A. 2(2014), p. 6086.

[9] Yang QH, Hou ZZ, Huang TZ: Journal of Applied Polymer Science. 132(2015), p. 41615.

[10] Jin Y, Huang S, Zhang M, et al: Synthetic Metals. 168(2013), p. 58. 